\title{
OPTIMALE VORAUSSETZUNGEN FÜR DIE ENTWICKLUNG DER REZEPTIVEN SPRACHFÄHIGKEITEN IM FRÜHEN FREMDSPRACHENUNTERRICHT
}

Im folgenden Beitrag wird die Antwort auf die Frage gesucht, nach welchen Prinzipien der frühe Fremdsprachenunterricht organisiert werden muss, damit er günstige Voraussetzungen für das rezeptive Lernen einer Fremdsprache schafft. Dabei wird von folgenden lerntheoretischen Prämissen ausgegangen:

1. Die Nativisten nehmen an, dass die Fähigkeit zum Sprachenlernen angeboren ist. Somit verfügt jedes Kind über einen natürlichen Spracherwerbsmechanismus (Chomsky 1957). Die natürlichen Spracherwerbsfähigkeiten werden durch einen Input aktiviert, der an Lernende herangetragen wird (Weskamp 2003: 25).

2. Damit aber die Lernenden ihre, ,intuitiven bedeutungsproduzierenden Mechanismen" optimal einsetzen können, muss ihnen ein unterstützender Input angeboten werden (de Cillia 1995: 210). Krashen (1985) ist in seiner Inputhypothese davon ausgegangen, dass eine Fremdsprache allein rezeptiv durch sprachlichen Input erworben werden kann, wenn dieser für die Lernenden verständlich ist (comprehensible input). Darüber hinaus sollte ein nach Krashen (1985) optimaler Input folgende Voraussetzungen erfüllen (in Vogel 1993: 157):

- „Er darf keiner grammatischen Progression folgen;

- er muss für die Lernenden interessant und relevant sein;

- er muss im ausreichenden Maße vorhanden sein."

Der Forderung nach Verständlichkeit kommt im Fremdsprachenunterricht eine zentrale Rolle zu, da der Input nur dann erworben werden kann, wenn er verständlich ist (Vogel 1993: 157). Wird den Lernenden dagegen ein incomprehensible input angeboten, so ist damit zu rechnen, dass die Informationsverarbeitungsprozesse entweder völlig blockiert oder überhaupt nicht in Gang gesetzt werden (Iluk 2005: 167). 
Der Anforderung, dass der Input für die Kinder interessant und relevant sein muss, stimmt Iluk (2005: 171) durchaus zu. Er meint, dass inhaltlich interessante Lernmaterialien, die die emotionale Seite des Lerners ansprechen, die Informationsaufnahme und -verarbeitung wesentlich unterstützen. Der ,affektive Filter“ (Krashen 1981) ist dann weit geöffnet und blockiert den Fluss der Informationen zu den kognitiven Zentren, wo sie verarbeitet und behalten werden, nicht. Wenn dagegen den Kindern Inhalte dargeboten werden, die Monotonie und intellektuelle Unterforderung bewirken, wie z.B. fremdsprachige Etiketten für bekannte Begriffe ( a dog - two dogs, a book - two books etc.), so kann das zu völligem Desinteresse am Fremdsprachenunterricht oder sogar zum Lernabbruch führen (Krashen 1981).

Bleyhls (2003: 7) Worte fassen am besten zusammen, was einen optimal beschaffenen Input ausmacht:

Die Schulkinder sollten mit der L2 in ihrer ,natürlichen" Form konfrontiert werden und nicht mit einem reduzierten Input, aus dem alles herausgefiltert wurde, was noch nicht im Unterricht behandelt wurde - vorausgesetzt natürlich, es ist ihrem Alter angemessen und vermittelt Inhalte, die ihren Interessen entgegenkommen. Die Auswahl der 'bearbeitbaren' Strukturen innerhalb dieses Inputs kann den impliziten Lernvorgängen überlassen bleiben.

3. Der angeborene Spracherwerbsmechanismus wird nur dann entsprechend genutzt, wenn in der Anfangsphase des Spracherwerbs eine relativ lange „stille Periode" (Inkubationsphase) respektiert wird, in der der Lernende das Fremdsprachensystem durch hörendes Verstehen internalisiert und nur selten fremdsprachliche Äußerungen produziert (de Cillia 1995: 210). ${ }^{1}$ In dieser Phase sind die Kinder nicht passiv, sondern geistig höchst aktiv, da sie hochkomplexe Leistungen erbringen müssen. Zu nennen sind u.a. folgende Leistungen (Mindt, Schlüter 2003: 42):

- „die Erkennung bedeutungsunterscheidender Laute,

- die Erkennung von Wortgrenzen im Kontinuum des Lautstroms,

- die korrekte Interpretation von Tonhöhen und ihrer Abfolgen (z.B. bei der Entscheidung über Aussage oder Frage),

- die Zuordnung von Bedeutungen zu Wörtern und Wortfolgen,

- die korrekte Einordnung des Gehörten in den Sinnzusammenhang."

In der ersten Phase des Fremdsprachenerwerbs konzentrieren sich die Kinder also auf das Entschlüsseln der fremdsprachlichen Zeichen. Der Verstehensprozess läuft nach Bleyhl (2000: 14, 20) und Iluk (2002: 22) wie folgt ab: Basierend auf Informationen aus Kontext und Weltwissen stellt der Lerner eine Hypothese über die Bedeutung der gehörten Sprachstrukturen auf. Die Erschließung der Bedeutung erfolgt also in der Wechselwirkung von Erfahrung und Erwartung. Trifft die Erwartung zu, so wird die Hypothese bestätigt und in die interne Sprache (innere Sprache) eingebunden. Wenn dagegen die Erwartung nicht bestätigt wird, wird die Hypothe-

${ }^{1}$ Die Lernenden verarbeiten mental die neuen Sprachphänomene und bauen entsprechende innere Repräsentationen auf (Iluk 2005: 165). 
se modifiziert. Um die aufgestellten Hypothesen verifizieren zu können, müssen die Kinder umgehend ein Feedback erhalten, wodurch die bei ihnen ausgelösten Enkodierungsprozesse bestätigt werden können. Das Sprachsystem zu erschließen gelingt dem Lerner, wenn er auf jeder Sprachaspektebene, vom Laut bis zur syntaktischen Struktur, das „Prototypische herausfiltert“ (Bleyhl 2000: 14). Dies ist nach Bleyhl (2000: 14) allerdings nicht möglich, wenn die Lernenden mit einem Sprachphänomen nur einmal in Berührung kommen.

Im sog. kommunikativen Anfangsunterricht mit seinem Stimulus-ResponsePrinzip wird der „stillen Periode“ keine Rechnung getragen, weil die Sprachproduktion von Anfang an forciert wird. ${ }^{2}$ Dem Kind gibt man somit keine Chance, den fremdsprachlichen Input hinreichend $\mathrm{zu}$ verstehen und eine mentale Repräsentation des neuen sprachlichen Systems zu entwickeln (Iluk 2005: 165f.). Durch eine solche Vorgehensweise kann keine Produktivität aufgebaut werden, denn „wir sind unfähig, für etwas, für das wir noch keine mentale Struktur, keine innere Repräsentation, kein inneres System ausgebildet haben, ein stabiles motorisches (Produktions-) Programm zu entwickeln" (Bleyhl 2000: 15).

4. Der Fremdsprachenunterricht in der Primarstufe besitzt ein Potenzial zur Förderung der rezeptiven Fähigkeiten der Grundschülerinnen und -schüler, sofern er auf jegliche strukturelle Progression verzichtet. Bleyhl (1996: 339ff.; 2000: 39) und Iluk (2002: 24f., 58f.) sind sich darin einig, dass fremde Sprachen in der Primarstufe nicht unter Beachtung der semantischen und grammatischen Progression (language syllabus) unterrichtet werden sollten. Im traditionellen Fremdsprachenunterricht, der dem Prinzip der Linearität folgt, wird ein Sprachphänomen nach dem anderen segmentiert. Die jeweiligen Einzelphänomene werden sofort „gesichert, vorgesprochen, nachgesprochen, geübt". Dabei wird vergessen, dass jedes Sprachphänomen nur dann seine Leistungsfähigkeit hat, wenn es nicht isoliert, sondern zusammen mit anderen Formen unterrichtet wird. Die kontrastive Gegenüberstellung der Spracherscheinungen (die Phase des Vergleichens), die als „Inkubationsphase” bezeichnet wird, erfolgt allerdings nur während der Rezeptionsphase. Dieser Phase wird in einem linear konzipierten Fremdsprachenunterricht zu wenig Zeit eingeräumt, weil die Schüler gleich zur Produktion der Sprachphänomene animiert werden. Darüber hinaus wird in einem linearen Fremdsprachenunterricht zu wenig Stoff, zu wenig Sprache geboten, was die Entstehung und Entwicklung mentaler Repräsentationen (mental chunks) des sprachlichen Systems verhindert. An Lehrbüchern, die der Progression nach semantischen oder grammatischen Gesichtspunkten folgen, ist zu bemängeln, dass sie oft nicht von Anfang an dem Kriterium der „kritischen Masse”

${ }^{2}$ Die imitativ-reaktive Lernweise wurde von Behavioristen entwickelt. Aus behavioristischer Sicht beruht der Spracherwerb auf Imitation der Sprache sowie Ausbildung und Automatisierung erwünschter sprachlicher Reaktionen (Weskamp 2003: 25). Diese Lernweise ist nach Ansicht vieler methodischer Autoritäten abzulehnen, denn ein Kind kann eine Sprache nicht effektiv lernen, wenn es wie ein Automat bestimmte Wendungen in immer gleichen Kontexten wiederholen muss, ohne das Gelernte kreativ auf andere Situationen übertragen zu können (Iluk 2002: 21f.; Mindt 2002: 5). 
gerecht werden. ${ }^{3}$ Die in den Lehrplänen vorgenommene Vokabelreduktion erweist sich im Fremdsprachenunterricht als kontraproduktiv (Bleyhl 2000: 16, 38).

In seinen Ausführungen bezieht sich Bleyhl (2000: 39) auf interessante Forschungsergebnisse aus Südostasien, von den Fiji-Inseln und aus Kanada (Elley, Mangubhai 1983; Elley 1991; Lightbown 1992), die die Effizienz eines nichtlinearen Fremdsprachenunterrichts bestätigt haben. Den Kindern wurden von den Lehrkräften Bilderbücher vorgelesen oder solche zum Selberansehen zusammen mit Tonkassetten zur Verfügung gestellt. Nach drei Jahren waren solche Klassen, deren Unterricht an Bilderbüchern orientiert war, den traditionell unterrichteten Klassen überlegen. Auch die Ergebnisse eines an deutschen Schulen durchgeführten Unterrichtsversuchs haben die Überlegenheit eines nicht linearen Unterrichts in fremden Sprachen empirisch abgesichert (Bleyhl 1996: 344f.; 2002: 39f.). ${ }^{4}$ Unterricht, in dem das von Winitz (1981) ursprünglich für den Selbstlernunterricht konzipierte Lehrmaterial Learnables benutzt wurde, brachte bessere Ergebnisse als ein lehrbuchorientierter Unterricht. ${ }^{5}$ Während des ganzen Schuljahres (40 Wochen) haben die Schüler etwa 1100 bis 1200 Wörter, einschließlich grammatischer Grundstrukturen, verstehen gelernt. Learnables wurde auch im Fremdsprachenunterricht mit Grundschulkindern ausprobiert (Bleyhl 2000: 39). Es hat sich gezeigt, dass zwar Grundschüler höherer Klassenstufen, im Vergleich mit Erstklässlern, bei denen die Konzentrationsspanne noch begrenzt ist, besser mit der Bewältigung der Aufgaben zurechtkamen, aber in allen Klassen die Entwicklung einer mentalen Selbständigkeit zu beobachten war. Dies lässt darauf schließen, dass diese Lerner später souveräner mit unbekannten Texten umgehen werden.

Die Überlegenheit eines nicht-linearen Fremdsprachenunterrichts liegt nach Bleyhl (1996: 346) darin, dass viel mehr Sprache umgesetzt wird. Somit werden „Wortschatz und Strukturen in immer verschiedenen Situationen erfahren und damit vielfältiger vernetzt und behalten". Dieses Sprachbad lässt sich nach Iluk (2002: 57f.) im frühen Fremdsprachenlernen hervorragend durch einen intensiven Einsatz des narrativen Ansatzes (story telling apporach) erreichen, der keiner semantischen oder grammatischen Progression folgt. Dafür liegen empirische Beweise vor.

Ergebnisse empirischer Untersuchungen, die in 90er Jahren in Italien durchgeführt wurden, liefern Beweise dafür, dass der Einsatz eines narrativen Ansatzes, der mit Geschichtenerzählen, Rollenspielen, Theater und anderen Aktivitäten adaptiert

${ }^{3}$ Es bedarf eines Wortschatzes von etwa 400 Lexemen bzw. unanalysierten ganzheitlich erfassten Wendungen, ehe der Lerner beginnt, selbst syntaktische Produktionen vorzunehmen (Marchman und Bates 1994 nach Bleyhl 2000: 16).

${ }^{4}$ Der Unterrichtsversuch wurde in einer Hauptschulklasse, zwei Realschulklassen und einer Gymnasialklasse im ersten Lernjahr Englisch als erste Fremdsprache durchgeführt.

${ }^{5}$ Jede Woche wurde eine Lektion mit 100 Sätzchen jeweils zu einem Bild vom Band vorgespielt bzw. gezeigt. Dies dauerte etwa 15 bis 20 Minuten. Am Ende jeder zweiten Lektion wurde ein Verstehenstest durchgeführt, bei dem die Schüler bei einer Bildserie jeweils angeben mussten, welchem von drei gezeigten Bildern ein vom Band gesprochener Satz entsprach. 
wurde, bessere Ergebnisse im Vergleich zu konventionelleren Methoden erzielte (Blondin 1998: 48). In einem der Projekte nannte man den starken fremdsprachlichen Input seitens des Lehrers als einen wichtigen Faktor, der zu besseren Resultaten im Erwerb der Fremdsprache beigetragen hat. Eine andere Untersuchung ergab, dass die Ausdrucksweise der Lernenden, die nach einem narrativen Ansatz unterrichtet wurden, mit dem typischen telegrafischen Stil kleinerer Kinder in ihrer Erstsprache vergleichbar war, d.h. Artikel und Präpositionen wurden in einer weiten Vielfalt ausgelassen. Die Kinder der Versuchsgruppe konnten allerdings Satzglieder und einfache Sätze bilden, auch wenn sie manchmal mit Fehlern behaftet waren. Am Ende der fünften Klasse waren sie sogar im Stande, kurze Geschichten zu erzählen, wobei sie neben- und untergeordnete Sätze verwendeten. Die Kinder aus der Vergleichsgruppe bildeten zwar korrekte, auswendig gelernte Sätze, viele jedoch vom Typ: This is... . Am Ende der fünften Klasse konnten die Kinder dieser Gruppe keine Geschichten erzählen.

Weitere empirische Beweise zur Effizienz eines nicht-linearen frühen Deutschunterrichts bietet eine Untersuchung, die in den Schuljahren 2003/2004 und 2004/2005 an einer Grundschule in Polen durchgeführt wurde (Gładysz 2007). Wie die Ergebnisse dieser Untersuchung zeigten, verfügten Primarstufenkinder, denen narrative Texte erzählt wurden, über einen größeren und differenzierteren rezeptiven Wortschatz im Vergleich zu den Grundschulkindern, die lehrbuchorientiert unterrichtet wurden. ${ }^{6}$ Die letzteren erhielten keine Gelegenheit, die fremde Sprache ganzheitlich zu erleben, weil ihnen ,reduzierte” Sprache, einzelne Wörter oder zusammenhanglose Einzelheiten angeboten wurden. Außer Gedichten, Reimen oder Liedern, die im Lehrbuch vorzufinden sind, wurde die Sprache in keinem sinnvollen Kontext angesiedelt. Bei der Präsentation der Freizeitaktivitäten wurden z.B. verschiedene Tätigkeiten mittels einfacher Sätze aufgezählt:

Sie schaukeln. Sie tauchen. Sie singen. Sie fahren Rad. Sie springen Seil ...

Das Gleiche war bei Winteraktivitäten zu beobachten:

Ich fahre Schi. Ich laufe Schlittschuh. Ich rodle gern ...

Das Antworten auf die Frage:

Was ist grün? Das Krokodil. Was ist braun? etc.

ergab für die Kinder wenig Sinn. ${ }^{9}$

${ }^{6}$ L. Halej, M. Kozubska, E. Krawczyk, Eins, zwei, drei. Podręcznik do języka niemieckiego dla klasy I (II i III). Warszawa: Wydawnictwo Szkolne PWN, 2000, 2003.

${ }^{7}$ Vgl. L. Halej, M. Kozubska, E. Krawczyk, Eins, zwei, drei. Podręcznik do języka niemieckiego dla klasy III. Warszawa: Wydawnictwo Szkolne PWN, 2000, 8f.

${ }^{8}$ Ebenda, 17.

${ }^{9}$ Vgl. L. Halej, M. Kozubska, E. Krawczyk, Eins, zwei, drei. Podręcznik do języka niemieckiego dla klasy II. Warszawa: Wydawnictwo Szkolne PWN, 2003, 8. 
Der extrem beschränkte Input, der zudem in keinem sinnvollen Zusammenhang angeboten wird, hat zur Folge, dass Kinder in der Entstehung und Entwicklung mentaler Repräsentationen (mental chunks) des sprachlichen Systems beeinträchtigt werden (Iluk 2002: 59). An den von Kindern der Kontrollgruppen erreichten Mittelwerten wird deutlich, dass die Kinder sogar das lexikalische Minimum nur unzulänglich beherrschten. Dies ist allerdings nicht verwunderlich, wenn man das geringe Stundenpensum (eine Stunde wöchentlich) sowie die Art und Weise der Präsentation von Sprachmaterial in Betracht zieht. Anhand der Unterrichtsbeobachtungen war noch anzumerken, dass Kinder von Liedern, Gedichten oder Reimen, die in dem verwendeten Lehrbuch vorhanden sind, sprachlich nicht zu viel profitieren konnten. Die Kinder konnten zwar den Text eines Liedes singen oder vorlesen, aber in vielen Fällen war ihnen die Bedeutung seines Inhaltes fremd. Die Ursache hierfür ist darin zu sehen, dass die Präsentationsphase zu kurz war und die Kinder gleich zum Nachsprechen animiert wurden, ohne den Inhalt zu verstehen (Gładysz 2007: 221f.).

Die Versuchsgruppe, die einen am narrativen Ansatz basierenden Deutschunterricht erhielt, hob sich auch hinsichtlich der rezeptiven Beherrschung grammatischer Strukturen von allen Vergleichsgruppen merklich ab. Dieser Effekt ist auf den intensiven Input zurückzuführen, der durch den story telling approach in einem nicht-linearen Prozess des Fremdsprachenlernens vermittelt wird. Zum Herausbilden von Mustern ist nämlich eine Speicherung von Daten erforderlich, die mehrmals in unterschiedlichen Kontexten zum Vorschein kommen. Durch die Auseinandersetzung mit der Sprache in ihrem ganzen Reichtum lernten Kinder, die einen am story telling orientierten Unterricht erhielten, längere und komplexere grammatische Strukturen zu verstehen als Schüler der Vergleichsgruppen. Die Kinder der Versuchsgruppe wurden nicht nur mit einfachen, sondern auch mit recht komplexen Sätzen konfrontiert, die große Differenziertheit im Hinblick auf ihre Verbindungen aufweisen. Sie bekamen auch die Möglichkeit, sich mit den Vergangenheitsformen vertraut zu machen, was den Kindern, die lehrbuchorientiert unterrichtet wurden, völlig vorenthalten wurde. Im Deutschunterricht, der auf die Arbeit mit dem Lehrwerk ausgerichtet war, wurden den Kindern grammatische Strukturen nur sehr vorsichtig dosiert. Dies hatte zur Folge, dass Kinder fast ausschließlich einfache Sätze zu hören bekamen, in denen z.B. auf die Deklination oder Steigerung der Adjektive verzichtet wurde. Überdies wurden alle Inhalte den Kindern im Präsens vermittelt. So eine Vorgehensweise verhindert das Herausbilden der oben erwähnten Muster grammatischer Strukturen (Gładysz 2007: 222ff.).

Das Hauptergebnis der Untersuchung muss auch im Zusammenhang mit der Einstellung der Kinder gesehen werden, denen Geschichten und Märchen erzählt wurden. Diese war, wie es während der Untersuchung beobachtet werden konnte, durchaus positiv. Eine solche Einstellung leistete zugleich einen Beitrag zum positiven Lernklima, das von Neugier, hoher Motivation und Lernbereitschaft getragen wurde. 
Abschließend ist allerdings zu bedenken, dass die Entwicklung der rezeptiven Sprachfähigkeiten der Kinder mit Hilfe des narrativen Ansatzes von einigen Bedingungen abhängig ist. Damit sich der Aufwand bezahl macht, der mit der Umsetzung der Storytelling-Unterrichtskonzeption zusammenhängt, müssen folgende Bedingungen erfüllt werden:

- Die an den meisten Grundschulen zur Verfügung stehende Unterrichtszeit von einer Unterrichtsstunde pro Woche ist erheblich unzureichend für eine effiziente Umsetzung des story telling approach. Iluk (2002: 28) vertritt sogar den Standpunkt, dass die Einführung des frühen Fremdsprachenunterrichts, der pro Woche nur einstündig erteilt wird, völlig verantwortungslos ist. Daher sollte eine Erhöhung der Wochenstundenzahl angestrebt werden. Nach Möglichkeit sollte der Unterricht in kurzen Einheiten gleichmäßig über die Woche verteilt erfolgen, weil die kindliche Konzentrations- sowie Erinnerungsfähigkeit ihre Grenzen hat.

- Wichtige Voraussetzungen für einen effizienten Einsatz des story telling approach sind auch eine entsprechende sprachliche und didaktische Kompetenz sowie Engagement seitens der Lehrkräfte. Der narrative Ansatz zeigt nur dann den erwarteten Effekt, wenn methodisch richtig vorgegangen wird. Um den Lehrkräften den zeitlichen Aufwand, der in die Umsetzung von narrativen Texten investiert werden muss, zu ersparen, wäre es wünschenswert, die Lehrenden mit fertigen Materialien zu versorgen. Anzustreben wäre die Herausgabe eines stufenübergreifenden Lehrwerkes, in dem narrative Texte in einer durchdachten Reihenfolge zusammengestellt würden.

\section{Literatur}

Bleyhl, W. (1996): Der Fallstrick des traditionellen Lehrens und Lernens fremder Sprachen. Vom Unterschied zwischen linearem und nicht-linearem Fremdsprachenunterricht. In: Praxis des neusprachlichen Unterrichts 4, 339-347

Bleyhl, W. (2003): Grammatikunterricht in der Grundschule? In: Fremdsprachen Frühbeginn (FF) 4, 5-9

Bleyhl, W. (2000): Fremdsprachen in der Grundschule. Grundlagen und Praxisbeispiele. Hannover: Schroedel

Bleyhl, W. (2002): Fremdsprachen in der Grundschule. Geschichten erzählen im Anfangsunterricht - Storytelling. Hannover: Schroedel

Blondin, Ch. u.a. (1998): Fremdsprachen für die Kinder Europas. Ergebnisse und Empfehlungen der Forschung. Berlin: Cornelsen

Chomsky, N. (1957): Syntactic Structures. The Hague: Mouton

de Cillia, R. (1995): Progression im Fremdsprachenunterricht. In: A. Carli (Hg.), Zweitsprachenlernen in einem mehrsprachigen Gebiet. Bozen, 205-217

Elley, W. (1991): Acquiring literacy in a second language: The effect of book-based programs. In: Language Learning 41: 3, 375-411

Elley, W.; Mangubhai, B.C. (1983): The impact of reading on second langugage learning. In: Reading Research Quarterly 19, 53-67 
Gładysz J. (2007): Empirische Untersuchung der Effizienz des narrativen Ansatzes. In: Orbis Linguarum 32, 205-230

Halej, L.; Kozubska, M.; Krawczyk, E. (2000, 2003): Eins, zwei, drei. Podręcznik do języka niemieckiego dla klasy I (II i III). Warszawa: Wydawnictwo Szkolne PWN

Iluk, J. (2002): Jak uczyć mate dzieci języków obcych? Katowice: Wydawnictwo Gnome

Iluk, J. (2005): Methodische Binsenweisheiten und der alltägliche Fremdsprachenunterricht. In: H. Pürschel, T. Tinnefeld (Hg.), Moderner Fremdsprachenerwerb zwischen Interkulturalität und Multimedia. Reflexionen und Anregungen aus Wissenschaft und Praxis. Bochum: AKSVerlag, 164-174

Krashen, S.D. (1981): Aptitude an attitude in relation to second language acquisition and learning. In: K.C. Diller (Hg.), Individual differences and universals in language learning aptitude. Rowley, Massachusetts: Newbury House, 155-175

Krashen, S.D. (1985): The Input Hypothesis: Issues and Implications. New York: Longman

Lightbown, P.M. (1992): Can they do it themselves? A comprehension-based ESL-Course for young children. In: Courchêne, u.a. (Hg.), Comprehension-Based Language Teaching: Current Trends. Ottawa: University of Ottawa Press, 353-370

Marchman, V.A.; Bates, E. (1994): Continuity in lexical and morphological development: a test of the critical mass hypothesis. In: Journal of Child Language 21, 339-366

Mindt, D.; Schlüter, N. (2003): Englisch in den Klassen 3 und 4. Grundlagen für einen ergebnisorientierten Unterricht. Berlin: Cornelsen

Vogel, K. (1993): Input und Fremdsprachenerwerb. Psycholinguistische Überlegungen zu Rolle und Funktion des sprachlichen Inputs beim Lernen und Verstehen einer Fremdsprache. In: Neusprachliche Mitteilungen (NM) 3, 151-162

Weskamp, R. (2003): Fremdsprachenunterricht entwickeln. Grundschule - Sekundarstufe IGymnasiale Oberstufe. Hannover: Schroedel

Winitz, H. (1981): Nonlinear learning and language teaching. In: The comprehension approach to foreign language instruction. Rowley, Mass.: Newbury, 1-13 